\title{
PRÁCTICAS DE LECTURA DE NOVELAS LITERARIAS EN ESTUDIANTES DE LA UNIVERSIDAD DE COSTA RICA
}

\section{READING PRACTICES OF LITERARY NOVELS IN STUDENTS OF THE UNIVERSITY OF COSTA RICA}

\author{
María Fernanda Fallas Rodríguez ${ }^{1}$ \\ mfernan2@cbu.edu \\ Rodrigo Muñoz González $z^{2}$ \\ rodrigo.munozgonzalez@ucr.ac.cr \\ Sergio Ulloa Garita $^{3}$ \\ sergioulloag@gmail.com \\ Luis Fernando Vargas Vega \\ luisdreamrush@gmail.com
}

\begin{abstract}
Resumen
Las prácticas de lectura de jóvenes es un tema que no ha sido abordado desde una perspectiva académica en el ámbito costarricense. Este artículo es una aproximación descriptiva de las prácticas de lectura de novelas literarias de los estudiantes de grado de la sede Rodrigo Facio Brenes de la Universidad de Costa Rica. Al estar inmersos en un ámbito académico, se eligió a esta población como sujetos de estudio. Por eso, se le aplicó encuestas a 121 estudiantes de diferentes facultades para indagar en sus prácticas de lecturas de novelas literarias, además se procuró detallar el formato y frecuencia de los y las estudiantes universitarios; así cómo identificar las novelas, géneros literarios y temáticas más populares. También, se precisó indagar el modo de adquisición de novelas literarias y la cantidad de tiempo de lectura dedicado a la lectura de estas.

Palabras clave: prácticas, lectura, estudiantes, universitarios, novelas, literarias.
\end{abstract}

\section{Abstract}

The reading practices of young people has not been analized from an academic perspective in the costarrican ambit. This article is a descriptive aproximation of the reading practices of literary novels of grade students of the Rodrigo Facio Campus of the University of Costa Rica. This poblation was chosen because they are immersed in an academic world. A survey was applied to 121 students from different faculties in order to inquire the format, frequency and schedule of reading of the university students;

1 Estudiante Universidad Christian Brothers.

2 Estudiante, Escuela de Ciencias de la Comunicación Colectiva, Sede Rodrigo Facio, Universidad de Costa Rica.

3 Estudiante, Escuela Dirección de Empresas, Sede Rodrigo Facio, Universidad de Costa Rica.

$4 \quad$ Colaborador de RedCultura.com 
thus, to identify the most popular novels, genres and themes. Also, the acquisition mode of novels and the quantity of time spent in reading were investigated.

Key words: reading, practices, university, students, literary, novels.

En Costa Rica, la lectura de textos tanto nacionales como extranjeros es promovida desde la educación primaria, y se da una publicación y promoción de obras literarias de autores costarricenses por parte de diferentes editoriales en el país.

Ante este panorama, resulta sensato realizar un estudio de las prácticas de lectura de los jóvenes costarricenses. Con un mayor conocimiento de las características y hábitos de los lectores jóvenes del país, se puede obtener información que permita una comprensión más integral del comportamiento de estos.

Sin embargo, en el ámbito nacional no se ha llevado a cabo ninguna investigación que aborde las prácticas de lectura de jóvenes costarricenses escolares, colegiales o universitarios. Por esto, resulta imprescindible empezar a adentrarse en el tema ya que los resultados de las investigaciones orientadas hacia las prácticas de lectura de jóvenes benefician a las instituciones educativas al darles una base para estructurar sus programas de estudio, y a las librerías y editoriales al brindarles información de sus consumidores.

La presente investigación, a través de un abordaje descriptivo, pretende delinear las prácticas de lectura de novelas literarias de los y las estudiantes de grado de la sede Rodrigo Facio de la Universidad de Costa Rica. Se eligió esta población porque se consideró que el estudiantado universitario, al estar envuelto en un ámbito académico, tiende a mostrar un mayor interés por la lectura de novelas literarias. Además, la población estudiantil de la Universidad de Costa Rica resulta conveniente para este trabajo ya que engloba jóvenes de todas partes del país con criterios variados según su especialidad académica y zona de procedencia.

Se procuró detallar el formato, espacio, frecuencia y horario de lectura de los y las estudiantes universitarios; así cómo identificar las novelas, géneros literarios y temáticas más populares. También, se precisó indagar el modo de adquisición de novelas literarias y la cantidad de tiempo de lectura dedicado a la lectura de estas.

Este estudio abarca la realidad costarricense del hábito de lectura de novelas literarias desde una aproximación de los Estudios Culturales con el propósito de contribuir al crecimiento de la discusión y conocimiento académico del tema. El impacto potencial de esta investigación radica en ser un punto de partida para futuras investigaciones que profundicen el tema y generen estudios de mayor alcance sobre la lectura a nivel nacional.

\section{Antecedentes}

Las Ciencias Sociales han evidenciado un interés particular por el estudio de las prácticas de lectura en la sociedad contemporánea. Se puede encontrar una gran variedad de investigaciones desde diferentes disciplinas y metodologías académicas que hacen extensa la bibliografía referente.

Enfocando el panorama a Latinoamérica y a España, son varios los estudios encargados de analizar las prácticas de lectura de los niños en etapa escolar y adolescentes que cursan la secundaria, destacando los estudios de Olaziregi (2000), Vilchez (2003), Domínguez (2005), Peredo y González (2007), Peña, Serrano y Aguirre (2008), y Contreras (2008). Por otra parte, la población universitaria también ha sido objeto de análisis en trabajos como los de Alterio y Pérez (2004), Belinche, Mancini, Díaz, Juárez y Altamirano (2007), y Nava Gómez y García Ávila (2009).

La investigación académica en Costa Rica no ha presentado estudios significativos acerca del tema en los últimos años, dejando un vacío de conocimiento y la necesidad de empezar a explorar las prácticas de lectura de la sociedad costarricense. Esta necesidad se vio reflejada en la iniciativa del Ministerio de Cultura y Juventud al realizar la Primera Encuesta de Hábitos 
y Prácticas Culturales durante los años 2010 y 2011 (Montilla y Avalos, 2012). En esta encuesta, $59.2 \%$ de las personas encuestadas dijeron no haber comprado un libro en el último año (Montilla y Avalos, 2012). Ante la misma pregunta, "el $23.5 \%$ con nivel universitario dijeron que había comprado de 1 a 3 libros" (Montilla y Avalos, 2012, p. 159). Así, se aprecia un mayor proclive de los individuos con educación superior a adquirir libros; sin embargo, la encuesta no se adentra en si estos textos corresponden a novelas literarias, cuentos, poesía o literatura especializada, abriendo la posibilidad de futuras indagaciones.

En general, la mayor parte de las investigaciones indican que la lectura en los grupos estudiados se da más por obligación que por iniciativa propia; en este sentido, la lectura académica es mayor que la lectura realizada por el disfrute personal. Como concluye el estudio de Peña, Serrano y Aguirre (2008), la lectura para los niños de la muestra analizada se encuentra asociada con un carácter pedagógico, en lugar de uno lúdico. Por otra parte, Nava y García (2009) muestran en su estudio que la población universitaria parece preferir invertir su dinero y su tiempo en amigos y otras actividades distintas a la lectura; y su interés por la actividad se reduce a la lectura de textos específicos para sus cursos universitarios.

$\mathrm{Si}$ bien los estudios mencionados anteriormente pertenecen a diferentes áreas de las Ciencias Sociales, se realizaron con diversidad de metodologías y fueron analizados desde diferentes perspectivas teóricas, todos coinciden en que la lectura es una práctica socio-cultural determinada por el contexto en que se desenvuelve cada individuo. Esto facilita que las prácticas de lectura puedan ser estudiadas como un fenómeno social que abarca poblaciones con ciertos rasgos de semejanza.

\section{Marco teórico}

Para desarrollar esta investigación, se tomó como base fundamental la Teoría de las Prácticas Culturales planteada por Pierre Bourdieu (2007) y el desarrollo del concepto de las prácticas de lectura por parte de Roger Chartier (1992).

Abordar la lectura desde la concepción de Bourdieu (2007) permite entenderla como una práctica social en la cual influye una lógica y una gramática específica del cómo se lee, una serie de determinantes culturales que configuran aquello que se lee y el para qué se lee. Mientras que los desarrollos de Chartier (1992) permiten identificar cuáles son las lógicas que son necesarias de analizar para poder comprender cómo se lee un texto en un contexto determinado.

Se partirá, para el presenta trabajo, que la lectura es una práctica cultural; estas prácticas surgen de los que Bourdieu (2007) denominó habitus, definido como

"sistemas de disposiciones duraderas y transferibles, estructuras estructuradas predispuestas a funcionar como estructuras estructurantes, es decir, como principios generadores y organizadores de prácticas y de representaciones que pueden ser objetivamente adaptadas a su meta sin suponer el propósito consciente de ciertos fines ni el dominio expreso de las operaciones necesarias para alcanzarlos, objetivamente 'reguladas' y 'regulares' sin ser para nada el producto de la obediencia a determinadas reglas, y, por todo ello, colectivamente orquestadas sin ser el producto de la acción organizadora de un director de orquesta" (p.86).

Esto tilda al habitus de ser normativo, estandarizador y regulador. Pero no es impuesto coercitivamente debido a la obediencia a un agente social con más poder, ya sea un líder de opinión o un líder político. Así, se determina que el habitus surge en la dinámica social como estructurante de la interacción cotidiana y es adoptado por los agentes sociales para el funcionamiento de la estructura total. Bourdieu (2007) ejecuta la metáfora de una orquesta que no posee conductor, lo cuál significa que el habitus funciona armónicamente entre sí, como una red en la cuál los agentes sociales pueden comunicarse intersubjetivamente $\mathrm{y}$, así, funcionar de manera integral.

A la vez, el habitus es producido por condicionamientos que produce una clase particular de condiciones de existencia (Bourdieu, 2007); es decir, el habitus de un determinado lugar depende, irremediablemente, de las características específicas del contexto cultural e histórico del mismo. En este sentido, el habitus como organizador de prácticas dependerá de las costumbres cotidianas de la zona, la forma en que los agentes sociales interactúen entre ellos y, en general, de la 
estructura social con la cuál funcione la dinámica social construida históricamente.

Bourdieu (2007) propone al habitus como la base del funcionamiento de las prácticas culturales, planteando, a su vez, las prácticas como normas a seguir de acuerdo con el habitus presente en el espacio social; de esta manera, las prácticas funcionan como manuales -los cuales van enfocados a fines específicos. Entonces, cualquier práctica cultural o social que se vaya a realizar tiene de antemano una serie de normas que dan una guía a seguir para realizar una acción. Tal es el caso de la lectura.

Las prácticas se encuentran relacionadas a instituciones las cuales regulan la forma en que se ejercerán, ya que la naturaleza de las mismas parece ser arbitraria (Bourdieu, 2007). Por ejemplo, la escuela sería la institución encargada de normar las prácticas de lectura debido a que es la organización encargada de la enseñanza y de desarrollar estas destrezas cognitivas por medio de diferentes factores que forman y determinan las prácticas de lectura.

Pero las prácticas y sus normativas no son del todo limitantes de la libertad de cada individuo. Hay una lucha entre las fuerzas exteriores e interiores de los cuerpos que, por medio de la interiorización de la externalidad, permite "a las fuerzas exteriores ejercerse, pero según la lógica específica de los organismos en los cuáles están incorporadas, es decir de manera duradera, sistemáticamente y no mecánica" (Bourdieu, 2007, p.89). Esto le da una libertad -limitada por los factores exteriores- a los individuos para ejercer la práctica de acuerdo con su historia personal o, como lo define el mismo Bourdieu (2007), singularidad de las trayectorias sociales. Entonces, “ el habitus que, en función de las estructuras producidas por las experiencias anteriores, estructura en cada momento las experiencias nuevas que afectan a esas estructuras en los límites definidos por su poder de selección" (Bourdieu, 2007, p.98).

De esta forma se puede entender que las prácticas están formadas por el habitus que norma, históricamente y por medio de las instituciones, las maneras en que se realiza una acción. Las prácticas se norman mediante habitus de clase o de grupo, los cuáles son familiares a todos los integrantes de la sociedad, pero no limitan completamente la libertad del individuo, el cuál puede ejercer cierto grado de libertad en el habitus gracias a su historia personal, o singularidad de su trayectoria social.

Roger Chartier (1992) ha dedicado gran parte de su trabajo al análisis histórico de las prácticas de lectura. El aporte de Chartier (1992) resulta importante debido a la capacidad que tiene su enfoque de establecer indicadores con los cuáles clasificar, para un mejor análisis, las prácticas de lectura. Los que resultan pertinentes para la presente investigación son: materialidad del texto, maneras de leer y creencias sobre la lectura (Chartier, 1992).

Para Chartier (1992), en concordancia con el planteamiento de prácticas de Bourdieu (2007), el leer es un proceso social e históricamente construido, realizado en un espacio intersubjetivo, " en el cuál los lectores comparten dispositivos, comportamientos, actitudes y significados culturales en torno al acto de leer" (Chartier, citado en Rockwell, 2001, p.14).

También, Chartier (1992) expone que la creación de sentido estará siempre dada por la interacción con el texto y las prácticas se generarán a partir de un encuentro entre el mundo textual -es decir, lo plasmado por el autor- y el proceso interpretativo del lector. Leer consta siempre de una práctica gestual, de espacios y de costumbres.

La lectura, vista como práctica cultural, se torna en un proceso intersubjetivo en donde el lector o lectora se ve inscrito en lo social y no está aislado de las demás personas lectoras y no lectoras con las que convive; la interacción con ellas modifica la manera en que lee un texto. Además, los y las lectores no son ajenos al bagaje histórico de la sociedad en que viven; tienen una formación histórica de la lectura, acerca de qué libros deben o no leer, la forma en que se lee, los espacios y tiempos en que se lee, el porqué se lee, el para qué se lee, qué concepción se tiene de los libros y el papel que juegan en la vida cotidiana de cada individuo. Todos los aspectos anteriores intervienen y modifican la ya subjetiva práctica que representa leer y apropiarse de los contenidos de los textos.

Chartier (citado en Rockwell, 2001) identifica la existencia de prácticas de lectura que 
amarran la manera en que se leía en cierto contexto; a esto lo llama maneras de leer, que es el primer indicador. En el caso pertinente de este estudio se refiere a dónde se lee, cómo se lee (si en voz alta o en silencio), los tiempos y los ritmos de lectura, y todo indicio que se pueda obtener sobre la manera en que la lectura forma o no parte del cotidiano de las personas. Es claro que al abarcar cómo se lee se debe preguntar primero quiénes leen.

Otra pregunta relevante es ¿en qué se lee?, esto hace referencia a la materialidad del texto, que resulta ser el segundo indicador. La materialidad se refiere a las características físicas del texto o libro en cuanto a "la disposición del texto en una página, la impresión y la encuadernación, el tamaño y la extensión del libro, su disponibilidad en determinados contextos y las huellas de su uso efectivo (Rockwell, 2001, p.15).

En el contexto de esta investigación, no solo hay que tomar en cuenta la materialidad en cuanto a texto impreso, sino los diferentes formatos en que se puede dar la lectura actualmente. Los formatos corresponden a libros en versiones impresas, en documentos digitales -ya sean txt, .doc o .pdf-libros electrónicos o E-books, la lectura por medio de lectores de libros digitales y tabletas, entre otros dispositivos y tipos de archivos que brindan las nuevas tecnologías con los cuales es posible realizar la lectura. Cuando se plantea la materialidad se debe proyectar la disponibilidad de los textos; es decir, la accesibilidad de los mismos, quiénes los tienen y en qué espacios es posible la lectura (Rockwell, 2001).

Ahora bien, ¿por qué se lee?, y ¿qué significa leer? Para Chartier (citado en Rockwell, 2001) resulta importante determinar las creencias sobre la lectura de cada época; estas presentan características peculiares sobre el significado que tiene la lectura para la sociedad, ya sea en el ámbito religioso -dónde la Biblia tiene la posición de libro sagrado y el leerlo involucra un ritual- o en la escuela y la academia dónde los libros son fuente de conocimiento.

Cada contexto le transmite un tipo de significado y de uso a los libros. Además, hay que pensar que leer cierto tipo de libros significa algo muy diferente que leer de otro tipo; es decir, cada tipo de libro que se lea tendrá un significado y uso particular dependiendo del ámbito sociocultural.

De esta manera, se entenderá como prácticas de lectura de novelas literarias a ese conjunto de prácticas y hábitos que hacen de la lectura un fenómeno social, construido y normado históricamente en el cuál intervienen la disponibilidad, el acceso, el formato, tiempos y espacios dónde se leen novelas literarias; además de la concepción de lectura y para qué se lee cada tipo de libros.

La comprensión de los géneros literarios es fundamental para el estudio de la novela literaria. El concepto de género puede utilizarse para referirse a varios niveles de la Literatura, desde la clasificación de la narrativa, la dramática y la lírica, a las subdivisiones de estas formas como la novela o la comedia, o incluso para hablar de una subdivisión: novela policiaca, romántica, infantil, entre otros (Spang, 2000).

Ya que esta investigación consiste en prácticas de lectura de novelas literarias, se puede tomar como base para elaborar una definición de género literario a las subdivisiones de esta forma y se adoptará el criterio temático y el lingüísticoenunciativo (Spang, 2000) como referencia. Así, para este trabajo se considerará que género literario se refiere a un grupo de novelas literarias que comparten rasgos estéticos, lingüísticos y temáticos similares.

Teniendo claro el concepto de género literario que se utilizará en este trabajo, se debe ahora considerar el término 'novela'. Villanueva (citado en Spang, 2000) propone que "la novela es el reino de la libertad, libertad de contenido y libertad de forma, y por naturaleza resulta ser proteica y abierta" (p.121); el amplio alcance de la novela obliga a delimitar sus concepto para favorecer esta investigación.

Una característica que diferencia a la novela de otras formas literarias es su extensión. "Desde principios del s. XVI la voz 'novela', derivada del italiano novella, se afincó para designar, primero la narración breve o mediana, y luego la extensa" (Spang, 2000, p.122). Esto permite definir como novela a la narración en prosa de larga extensión. Como el carácter de extensión es subjetivo y cambia dependiendo del lector, para este trabajo la extensión tendrá que superar las 50 páginas. 


\section{Metodología}

Esta investigación fue realizada en la sede Rodrigo Facio de la Universidad de Costa Rica debido a las facilidades que representa en cuanto a ubicación, tiempo y diversidad de muestra; con esto, se pretende describir las prácticas de lectura de novelas literarias de los estudiantes pertenecientes a las facultades de Ciencias Sociales, Educación, Bellas Artes, Letras, Ingeniería, Ciencias de la Salud y Ciencias Exactas.

Se partió de la hipótesis de que la mitad de los estudiantes de la población a analizar son lectores de novelas literarias de manera recurrente, ya sea en formato digital o impreso, y que entre los títulos preferidos de los y las estudiantes se encuentran libros populares y de gran venta en la actualidad como la serie de Harry Potter y las novelas de Paulo Coelho, además de autores clásicos latinoamericanos.

La población que formó parte de esta investigación fueron estudiantes de grado de la sede universitaria Rodrigo Facio de la Universidad de Costa Rica. Se consideró que la frecuencia de la práctica de lectura de novelas literarias es mayor en la Gran Área Metropolitana (GAM) debido a factores como una mayor tendencia histórica al consumo de libros y el acceso limitado de librerías e Internet en zonas rurales; además, como se indicó anteriormente, se consideró que el estudiantado universitario conforma un grupo más involucrado en la lectura de novelas literarias debido a su contacto con el ámbito académico.

La muestra de la investigación está compuesta por un grupo de 121 estudiantes, dividido en dos áreas de estudio: ciencias teóricas y ciencias técnicas. La primera se refiere a aquellas disciplinas cuyo campo de estudio es el ser humano y la segunda a aquellas especializadas en el mejoramiento de técnicas. Cada uno de estos grupos fue dividido según la facultad a la que pertenecen, la cual a la vez se subdivide en ciertas carreras. El primer grupo cuenta con estudiantes de la Facultad de Ciencias Sociales (Comunicación Colectiva, Psicología, Antropología/Sociología y Ciencias Políticas), estudiantes de la Facultad de Educación (Educación Primaria, Educación Secundaria y Educación Preescolar), estudiantes de la Facultad de Bellas Artes (carreras musicales, Artes Plásticas y Artes Escénicas) y estudiantes de la Facultad de Letras (Filología, Filosofía e Inglés o Francés). El segundo grupo cuenta con estudiantes de la Facultad de Ingeniería (Ingeniería Civil, Ingeniería Industrial, Arquitectura y Informática), estudiantes de la Facultad de Salud (Medicina, Microbiología y Farmacia) y estudiantes de la Facultad de Ciencias Exactas (Física, Química, Biología y Matemáticas).

Como puede apreciarse, no se han incluido todas las carreras que ofrece la Universidad en vista de que son más de 50 y el tamaño de la población necesaria sería muy difícil de alcanzar, por lo que se eligieron aquellas consideradas las más populares y representativas de cada Facultad. Según información suministrada por la Oficina de Registro e Información de la UCR (s.f), la población de estudiantes regular matriculada en el primer semestre del 2011 era de 34 661, por lo que el tamaño de la muestra no proporciona una verdadera representatividad de la población estudiada. Pero debe recalcarse que el propósito de este trabajo es plantear un inicio descriptivo del tema y no realizar un estudio a nivel macro del tema.

La investigación optó por tres tipos de muestreo. Primero, se utilizó el muestreo estratificado no representativo, que consiste en la división de la población en estratos y selección de la muestra según cada división (Hernández, 2006). Los estratos delimitados en este caso corresponden a las facultades de Ingeniería, Ciencias de la Salud, y Ciencias Exactas para la categoría de las ciencias técnicas; y las facultades de Ciencias Sociales, Educación, Artes, y Letras para las ciencias teóricas. A su vez, dichas áreas se subdividen en carreras específicas para casa área.

Tomando en cuenta la naturaleza del universo en estudio, esta investigación utiliza las muestras de juicio; estas son "aquellas en las que el investigador 'arma' la muestra de tal modo que ésta refleje lo mejor posible al universo 'a su criterio' " (Davidovics y Mayol, 2008, p.77). Este tipo de muestra se utiliza basándose en la clasificación que se realizó. Por último, se utilizó el muestreo por conveniencia; esto se debe a que "estas muestras se caracterizan por seleccionar las unidades muestrales, personas u objetos, por su facilidad y cercanía” (Davidovics y Mayol, 2008, p.78). 
La presente investigación remitió a un par de retos a la hora de la recolección de datos. El primer reto se refería a la población de la Universidad; al ser una cifra muy alta, se debió escoger un mecanismo apropiado que permitiera el flujo de la investigación. El segundo tendría que ver con la correcta confección del mecanismo de medición. Se consideró que el método más adecuado para conseguirlo era la encuesta.

La encuesta se realizó por medio de un cuestionario de autoentrevista, donde el informante llenaba de manera independiente su cuestionario impreso, permitiendo un proceso de recolección de datos ágil. La versión final contó con diferentes modalidades de preguntas: abiertas, cerradas de selección única y múltiple, y de combinación de tipo de preguntas.

\section{Resultados y hallazgos}

Para facilitar una mayor claridad y una mejor comprensión, los resultados y hallazgos obtenidos de esta investigación se desarrollarán a los largo de cuatro capítulos.

\section{Capítulo 1: Características de la recolección de datos}

La recolección de datos se llevó a cabo durante siete días a lo largo del mes de octubre del año 2011; la recolección fue realizada en los días $14,17,18,19,20,25$ y 27 de octubre. Para la presente investigación, no se definió una cuota específica de género en la muestra inicial, pero se buscó garantizar una equidad en la selección; no se utilizó ningún mecanismo probabilístico para validar esta variable. Los resultados finales arrojaron que $49 \%$ de los encuestados eran hombres, un $46 \%$ eran mujeres y un 5\% se abstuvo de responder.

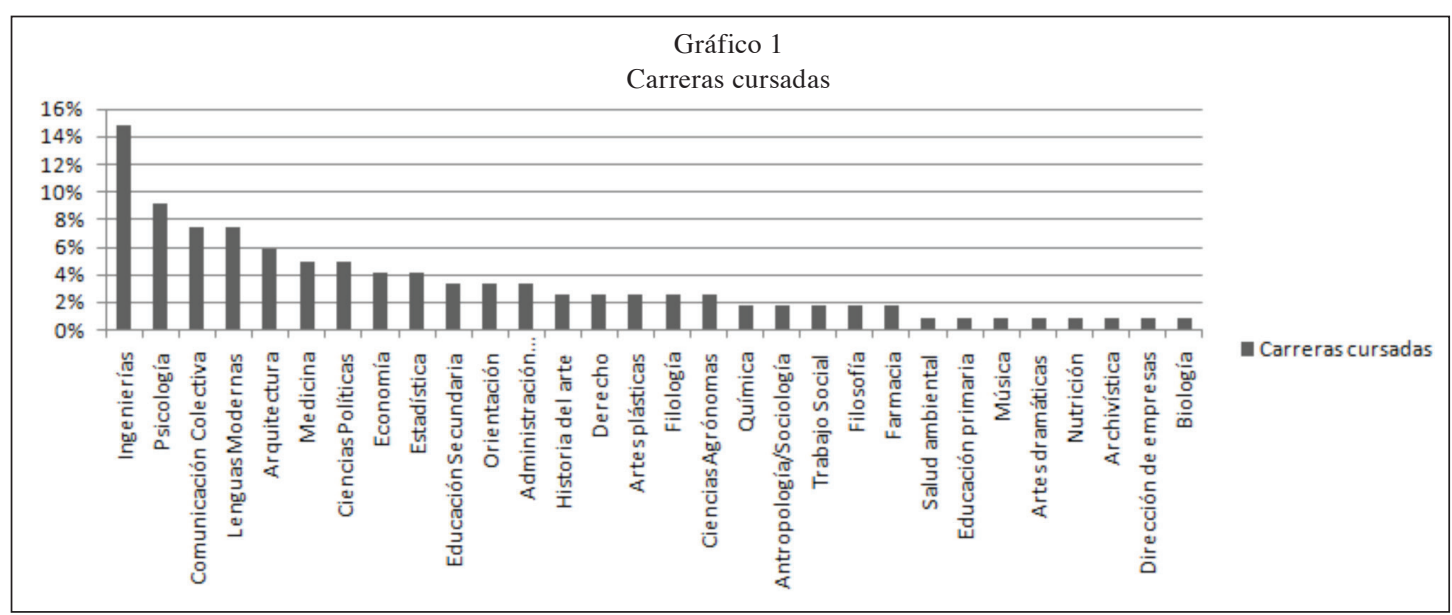

Fuente: Elaboración propia.

Para asegurar una representatividad 'justa', se trató de buscar una cifra de carreras que fuese similar con las características académicas fijadas en la muestra inicial. Sin embargo, la mayoría de personas entrevistadas cursa carreras pertenecientes a la Facultad de Ciencias Sociales.

\section{Capítulo 2: Frecuencia y formatos de lectura}

\section{Frecuencia}

Se buscó indagar el tiempo que los encuestados dedican a la lectura. Para ello, se preguntó 
sobre el tiempo de lectura semanal, que a su vez, se dividió en dos periodos: el tiempo lectivo y el de vacaciones. Los resultados indicaron que durante las vacaciones, para la mayoría de la muestra, el tiempo semanal destinado es menor a 6 horas. Es decir, no se dedica más de una hora diaria a la lectura. Esto puede atribuirse a la costumbre de muchas personas de entregarse intensivamente a una novela durante períodos cortos y después no volver a leer durante un período.

Durante el período lectivo los tiempos de lectura disminuyen como era de esperarse. Casi la mitad de la muestra dedica una hora o menos a la lectura de novelas literarias. Resulta interesante que el mayor porcentaje corresponde a dos horas en lugar de ninguna. Ya que el tiempo semanal difícilmente estará distribuido de forma equitativa a lo largo de la semana, es probable que estas dos horas o menos sean empleadas durante el fin de semana.

En resumen, los resultados indican que las personas leen más en vacaciones que en tiempo lectivo, lo cual podría atribuírsele a la cantidad de tiempo libre disponible para realizar la actividad. Sin embargo, se debe considerar que los tiempos de lectura de la media no varían mucho, en vacaciones un $40 \%$ afirmó leer menos de 4 horas semanales y un $20 \%$ dedicar entre 4 y 6 , mientras que en período lectivo un $30 \%$ afirmó dedicar dos horas semanales a la lectura de novelas literarias y un $27 \%$ dedicar menos de una hora. Esto indica que la mayor parte de los estudiantes no invierten más que unas cuantas horas a la semana en la lectura y es muy posible que no lean a diario; es decir, no invierte mucho tiempo de su cotidiano en la lectura.

A manera de complemento, se buscó indagar la cantidad de novelas literarias leídas anualmente. Con esto se descubrió que muchos de los lectores, especialmente los de gustos menos "comerciales", suelen leer más de una novela simultáneamente; como la pregunta generó cierta ambigüedad entre algunos encuestados, muchos no sabían si tomar en cuenta novelas que no habían logrado finalizar. En este caso, también se les solicitó que tomaran en cuenta aquellas novelas que se encontraban leyendo pero que no habían logrado finalizar. El promedio es de una a tres novelas en el año.
Las cifras indican que la mayor parte de los estudiantes leen pocas novelas anualmente, lo que va acorde a la cantidad de tiempo que dedican a la lectura, el cual es poco. Aunque un resultado que parece contrastar con este bajo número de novelas leídas por año es el de la pregunta ¿se encuentra leyendo alguna novela literaria actualmente? Pese a realizar la recolección de datos a final del semestre lectivo, período durante el cual la carga académica exige dedicar más tiempo a la universidad, un 53\% de la muestra se encuentra en medio de una novela literaria. La mayor parte afirmó avanzar lentamente en ella desde que inició el semestre.

De acuerdo con estos resultados, el hábito de lectura en los estudiantes parece ser una lectura lenta y a detalle, de pocos libros pero constante durante el año. Los estudiantes en lugar de leer constantemente y terminar la lectura de las novelas en poco tiempo, suelen tardar varios meses leyendo sus novelas.

La frecuencia de la lectura en los estudiantes de la muestra parece ser de pocas horas semanales pero constante durante el año, con una disminución a tomar en cuenta durante periodo lectivo. Los estudiantes parecen leer pocas novelas al año debido a que la leen durante tiempos extensos en vez de dedicar muchas horas semanales y terminar rápido.

\section{Formato}

El formato indica la consistencia del habitus instaurado en el contexto social, esto porque demuestra que a pesar de los cambios tecnológicos la norma instaurada socialmente puede prevalecer. Esto se traduce en que, a pesar de las facilidades que permiten los formatos digitales y su adquisición por Internet como la descarga gratuita, y a veces ilegal, y la rapidez de adquisición, las personas pueden preferir la lectura en papel porque es lo habitual, lo que se conoce y se practica desde hace siglos. Los resultados indican que el formato impreso sigue siendo el predilecto, especialmente por la reciente penetración de medios de lectura digital, los cuales apenas se encuentran estableciéndose en el país. Sin embargo, puede observarse que un número considerable utiliza ambos formatos, especialmente mediante 
la descarga de Internet. El 52\% de la muestra se inclinó por leer en el formato impreso y un $36 \%$ indicó usar los dos formatos. Esto da un total del $88 \%$ de la muestra que usa el formato impreso para realizar su lectura.

Además de esto, se preguntó el lugar de adquisición de las novelas literarias que leen. Esta pregunta permite obtener el modo de adquisición de novelas literarias por parte del estudiantado encuestado. La opción que fue elegida más ocasiones fue la de 'librerías', la cual obtuvo un 72,7\%. La opción de 'prestamos' ostentó el segundo lugar de elección con un 57,8\%. El tercer lugar de elección, con un 43\%, lo obtuvo la opción de 'bibliotecas'.

Esta pregunta demuestra que el estudiantado encuestado prefiere lugares tradicionales de adquisición de novelas como las librerías, las bibliotecas o el préstamo por parte de otras personas. Sin embargo, se nota una tendencia a un modo de adquisición que involucra nuevas tecnologías como Internet ya que un 39,7\% del estudiantado entrevistado eligió la descarga de novelas por Internet, y un $10,7 \%$ de los y las personas entrevistadas manifestó que adquiría las novelas literarias comprándolas en Internet. La difusión de formatos como los e-books y lectores digitales como el Kindle y el Nook, abren la posibilidad de abrir la incógnita acerca de la apropiación de los y las estudiantes de la Universidad de estos dispositivos.

Estos resultados terminan de probar que el formato impreso es el predominante, por encima del formato digital o dispositivos de lectura digitales. El libro impreso, ya sea por facilidad de lectura o por una condición socio-histórica tiene un lugar instaurado en las prácticas de lectura del estudiantado. Es muy posible que el contexto y la tradición mantengan al libro en su lugar predominante en la sociedad y lo mantenga así por varios años a venir.

\section{Capítulo 3: Géneros y temáticas preferidas}

Durante el proceso de encuesta se trató de indagar entre los participantes cuál era el género que más frecuentan entre sus prácticas de lectura de novelas literarias. Para esto, en la encuesta se les ofreció una lista que incluía los géneros más relevantes y representativos dentro de la literatura a criterio de los realizadores de esta investigación, con base en las fuentes consultadas (Spang, 2000). Se le permitió a la muestra marcar cuantos géneros desearan dentro de las nueve opciones disponibles, incluso todas si así lo deseaban. Es importante observar la apropiación que hacen los estudiantes sobre los géneros que frecuentan, pues es muy común que muchos no sepan a cuáles géneros pertenecen sus autores o novelas preferidas.

La lista de géneros a elegir incluían: policíaco, histórico, romántico, juvenil, espiritual, latinoamericano, ciencia ficción, fantástico e infantil. Los géneros elegidos en mayor número corresponden al de ciencia ficción y fantástico: estos representan un $55 \%$ y $53 \%$ del total de la muestra, respectivamente. La proximidad de los porcentajes sugiere que muchos lectores no perciben mayor diferencia entre el uno y el otro. Además, pese a ser los predilectos entre los encuestados, la saga de Harry Potter es el único libro entre los diez más leídos que pertenece completamente a alguno de estos géneros. La elección puede obedecer al bombardeo de títulos pertenecientes a estos géneros en los últimos años, como Twilight o la saga de Narnia; además, el interés ha crecido al realizarse múltiples adaptaciones cinematográficas de novelas pertenecientes a estos géneros.

Posteriormente se encuentran los géneros latinoamericano e histórico, en iguales proporciones de $50 \%$. Es decir, la mitad de los encuestados frecuenta leer novelas de este estilo. Títulos populares entre la muestra como Cien años de soledad y El amor en los tiempos del cólera confirman esta predilección. Nuevamente, surge la ambigüedad sobre qué consideran estas personas como género histórico, pues muchos consultaban si una novela de corte ficticia desarrollada en el pasado podía considerarse histórica. La interpretación personal de cada individuo dentro de los géneros juega un papel determinante en los resultados obtenidos.

Finalmente, el género romántico se ubicó en quinto lugar entre los más populares, con una diferencia significativa frente a los dos anteriores. Orgullo y Prejuicio fue la novela romántica más citada, en especial dentro de población femenina. Los géneros que cerraron la lista, en orden descendiente, fueron: policíaco, juvenil, espiritual e infantil. 


\section{Capítulo 4: Las novelas y los autores más leídos}

Las prácticas de lectura no se limitan al cómo leen los estudiantes, sino que incluyen el qué leen, ya que esto indica las tendencias que hay en los estudiantes de seguir el mercado y la publicidad en cuanto a libros o bien usar la lectura como una forma de expresión de su individualidad. El autor o la novela que lee cada quién dice algo de quiénes son y sus gustos, los constituye y exterioriza lo que les apasiona o interesa. Es por esto que se decidió indagar en las novelas y autores preferidos por los encuestados, en aras de observar si hay una tendencia de seguir la publicidad y el mercado, en otras palabras, los autores del momento o bien si había gustos variados y específicos.

Lamentablemente los resultados arrojaron más de 250 títulos de novelas y más de 300 autores, lo que es imposible de graficar. Debido a esto se decidió ilustrar las 10 novelas y autores más leídos del total.

\section{Novelas favoritas}

La primera pregunta referente a este capítulo en la encuesta era totalmente abierta y una de las de mayor interés dentro de la investigación corresponde a las cinco novelas preferidas de los encuestados. Entre las respuestas se contabilizaron 252 títulos distintos tan solo entre 121 personas, por lo que puede decirse que existe una variedad considerable de novelas dentro de la población universitaria encuestada. Para efectos prácticos, y dado que muchas de las novelas fueron mencionadas tan solo una vez, se muestran los 10 títulos más populares. La novela predilecta, por una considerable diferencia, es Cien años de soledad de Gabriel García Márquez, el cual también resultó ser el autor de mayor preferencia como se muestra más adelante. Las conocidas sagas de novelas más populares de los últimos años (Harry Potter, El señor de los anillos y Twilight) no tuvieron el peso esperado al cual se hizo referencia en la hipótesis de la investigación. Solo la primera hizo aparición en la lista de las 10 novelas más populares, respaldada por la elección de tomar cada libro de la saga como una misma opción. Vale la pena mencionar la mención de dos novelas costarricenses dentro de la lista: Los Peor y Viaje al reino de los deseos, en especial porque la primera no forma parte del plan de estudios en la secundaria.

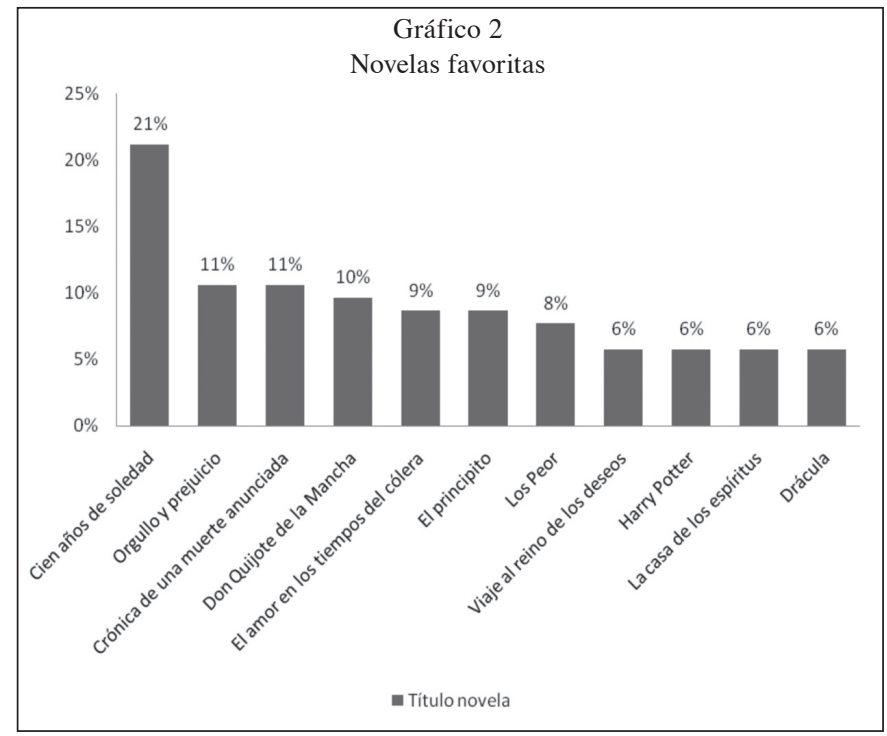

Fuente: elaboración propia. 
Esto indica que en gran parte el gusto de los estudiantes no atiende a los títulos populares del momento, ni sigue la tendencia de las novelas más publicitadas en el momento. Pese a que la saga de Crepúsculo y las novelas de Dan Brown no están entre las primeras diez, sí fueron mencionadas en la lista total.

$\mathrm{Al}$ parecer el gusto por novelas de los estudiantes gira en torno a sus intereses personales y la tradición literaria en el mercado. Se dice tradición literaria porque las novelas mencionadas se consideran clásicos de la literatura y están instauradas en el colectivo como novelas de alta calidad literaria.

\section{Autores preferidos}

Los autores indican si hay una preferencia de lectura basada en el mercado, o bien, por factores como gusto personal o tradición del contexto socio-cultural. Como se mencionó anteriormente, Gabriel García Márquez fue el autor de predilección entre la muestra, nuevamente con una diferencia importante. Fernando Contreras fue el único autor costarricense que aparece dentro de la lista, lo cual concuerda con la mención de su novela Los Peor dentro del gráfico anterior. Se recopiló un total de 330 autores entre la muestra. Sin embargo, este fue el espacio que quedó en blanco con más frecuencia entre todos los cuestionarios. Muchas personas no fueron capaces de responder quién era el autor de sus novelas favoritas.

Los autores indican una elección basada en gustos y tradición más que tendencias del mercado actual de venta de libros. Esto significa que los estudiantes tienen un criterio basado en su individualidad para elegir qué van a leer y a quién van a leer.

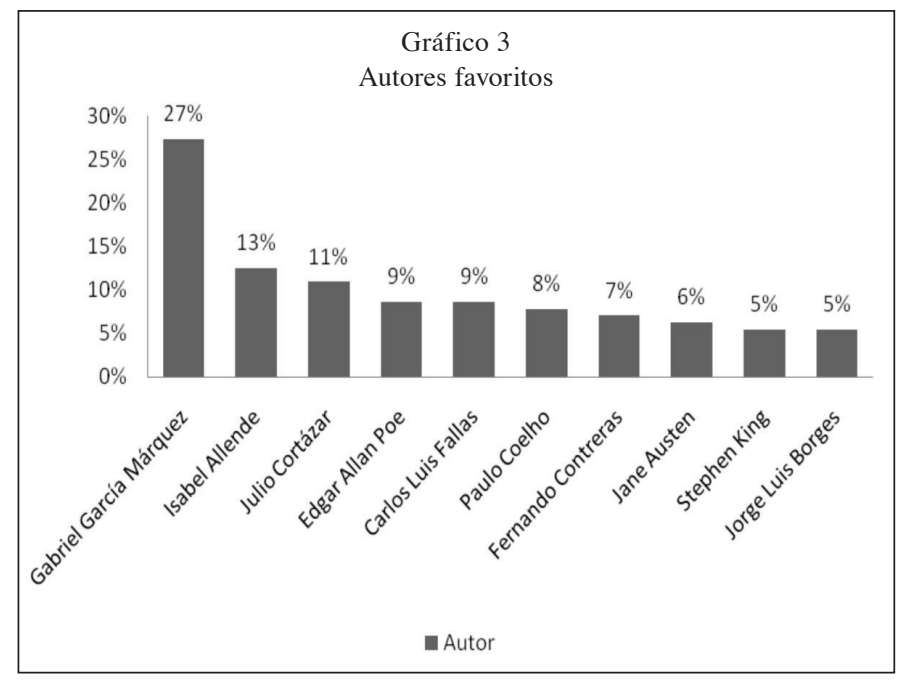

Fuente: elaboración propia.

\section{Consideraciones finales}

Los resultados finales de la investigación muestran de una manera bastante satisfactoria las prácticas de lectura de los estudiantes encuestados de la sede Rodrigo Facio de la Universidad de Costa Rica. Por lo que se puede decir que se consiguió alcanzar los objetivos de la investigación.

En primer lugar, se logró identificar cuáles eran las frecuencias y formatos que utilizaban los estudiantes de la muestra para leer sus novelas preferidas. Los resultados indican que la mayoría otorga poco tiempo a la lectura por semana: 
en vacaciones el $40 \%$ afirmó dedicar menos de 4 horas semanales, en periodo lectivo un $20 \%$ afirmó no destinar horas y un $27 \%$ indicó utilizar menos de una hora a la lectura de novelas literarias. Esto resulta en que los estudiantes disminuyen la cantidad de horas dedicadas a la lectura de novelas literarias cuando se encuentran en clases.

Por otra parte solo un $7 \%$ de los encuestados manifestó que no lee ninguna novela al año, y 53\% dijo estar leyendo una novela en el momento de que la encuesta fue aplicada. Además practican un tipo de lectura lenta y continua durante el año, esto debido a que leen en promedio pocas novelas -la mayoría de 1 a 3 - y lo hacen por tiempos cortos por semana, en general menos de 4 horas. Esto indica que los estudiantes leen en periodos cortos pero continuos, lo que significa que se toman mucho tiempo para leer una novela en vez de dedicar muchas horas semanales y terminar la lectura de manera rápida.

La tradición histórica e influencia de la misma en las prácticas de lectura se puede reflejar en el formato que escoge el estudiantado para leer. El formato preferido es el impreso, un 52\% dijo preferirlo y un $36 \%$ dijo combinarlo con lectura digital. Esto se puede entender como una tradición más que falta de recursos para obtener las novelas en digital, ya que la mayor parte de los estudiantes universitarios posee, de alguna u otra forma, acceso a Internet. También puede influir un asunto práctico, ya que la lectura en impreso es mucho menos cansada que la lectura en una pantalla.

Por otra parte se logró indagar en los géneros de novela que prefieren los estudiantes encuestados: los géneros preferidos son el de ciencia ficción y fantástico, además hay un gusto por las novelas latinoamericanas e históricas. Esto refleja especificidades en las preferencias de los estudiantes, reflejan sus gustos y personalidades, dicen algo sobre ellos.

Por último se logró identificar las novelas y autores preferidos por la muestra. Los resultados indican que clásicos de la literatura como Cien años de Soledad y El Principito son de los más gustados por los estudiantes, al igual se pueden encontrar novelas costarricenses en la lista. Esto más la gran cantidad de novelas que mencionaron los entrevistados indican que las preferencias de lectura no siguen al mercado y la publicidad del momento y más bien tienden a preferencias e intereses personales y a una tradición histórica de novelas consideradas clásicas.

En relación a la hipótesis planteada, se comprobó que efectivamente casi la mitad de estudiantes encuestados se consideran a sí mismos lectores habituales de novelas literarias. Sin embargo, la mayor parte de ellos tienen períodos que dedican a la lectura, especialmente durante vacaciones. De esta forma, puede decirse que su frecuencia gira en torno al momento lectivo en que encuentren, pues durante clases la mayor parte disminuye radicalmente o dejan a un lado la lectura.

En cuanto a las novelas que se plantearon como populares, la saga de Harry Potter no recibió tantas menciones como se esperaba originalmente, mientras que ningún libro de Paulo Coelho figuró en la lista de 10 novelas preferidas entre los lectores. Al contrario, se encontró una enorme variedad de gustos literarios entre la muestra, con más de 250 títulos mencionados, la mayor parte de ellos no más de una vez. La preferencia por autores latinoamericanos sí se cumplió en la forma esperada, con Gabriel García Márquez liderando la lista, además de otros autores de la región como Isabel Allende y costarricenses como Fernando Contreras.

Se puede decir que el espacio universitario puede influir en las elecciones de lectura. La universidad presenta un lugar particular en donde se discuten ciertos temas y el nivel de educación es elevado, esto puede influir en que los y las estudiantes rechacen lo que les vende el mercado y lo consideren como literatura light y se enfoquen en novelas con más contenido y tradición histórica.

Las prácticas de lectura de los jóvenes universitarios se ven en parte influenciadas por el contexto socio-cultural y la tradición histórica de la lectura pero a la vez hay mucha influencia de la trayectoria social individual de cada estudiante. Esto influye en especial en lo que lee el estudiante más que en cómo lo lee.

La principal faltante en esta investigación es lo pequeña y poco representativa que resulta su muestra frente a la población universitaria que pretende representar. Para una investigación a futuro, se incita a alcanzar un número 
considerable de estudiantes dentro de cada facultad, en una distribución similar a la planteada, pero con un número que alcance al menos los 5000 mil estudiantes. De todas formas, el objetivo inicial de esta investigación fue analizar y obtener conclusiones con base en la muestra estudiada, sin trascender a los estudiantes universitarios en general.

Al igual, la población de esta investigación se delimitó a estudiantes de grado de la sede Rodrigo Facio de la Universidad de Costa Rica; sin embargo, hubiese sido pertinente explorar el estudiantado de las sedes regionales. Esto permitiría realizar una comparación entre los hábitos de las distintas sedes de la universidad.

Por otra parte, dentro de la elaboración del cuestionario, algunas preguntas necesitan ser modificadas para eliminar la ambigüedad que experimentaron algunos de los encuestados. El factor común dentro de este problema es la percepción personal que cada persona pueda darle a términos como 'lector habitual' o si 'haber leído' una novela implica finalizarla. Si la encuesta pretende seguir aplicándose de este modo, sería mejor realizarla en forma de entrevista corta, pues como cuestionario autoadministrado no permite aclaraciones vitales para obtener la información necesaria. Otro ejemplo donde puede ilustrarse esta situación es la pregunta relacionada a novelas costarricenses que no pertenecieran al plan de estudios de la secundaria. Muchos de los encuestados no recordaban cuáles eran las novelas que pertenecían a este plan, y en otros casos los planes diferían entre colegios.

También, hubiese sido interesante para los análisis finales dejar un espacio en el instrumento de recolección de datos dónde el estudiantado encuestado tuviese la opción de marcar que no lee del todo.

Aunque la presente investigación estuvo enfocada en novelas literarias por cuestión de delimitar el tema, sería pertinente abrir el campo para desarrollar una investigación que se preocupe por realizar un mapeo acerca de las prácticas de lectura de los estudiantes de cualquier texto; así, se lograría medir no solo la lectura de literatura, sino las de las antologías pertinentes a cada curso.
Los autores de esta investigación quisieran expresar su eterno agradecimiento a la M.Sc. Yanet Martínez, a la M.Sc. Sonia de la Cruz, a la Dra. Patricia Vega y al Dr. Carlos Sandoval por todo el apoyo brindado a lo largo del proceso.

\section{Referencias bibliográficas}

Alterio, G. y Pérez, H. (2004). Hábitos de lectura en estudiantes universitarios. Revista Cubana de Educación Medica Superior, 18(1), 1-8. Obtenido de base de datos EBSCO Host el 19 de agosto del 2011.

Belinche, M., Mancini,G., Viñas, R., Díaz, C., Juárez, F. y Altamirano, L. (2007). Los jóvenes y la lectura. Estudio de las prácticas de lectura y de consumos de los ingresantes a la facultad de periodismo y comunicación durante los ciclos 2006 y 2007. Recuperado el 17 de agosto del 2011 de http://www.perio.unlp.edu.ar/ojs/index. php/question/article/viewFile/455/379

Bourdieu, P. (2007). El sentido práctico. Buenos Aires: Siglo XXI Editores.

Chartier, R. (1992). El Mundo como Representación: Estudios sobre la historia cultural. Barcelona: Editorial Gedisa.

Contreras, J. (2008). Leer en tiempos modernos: adolescentes y jóvenes profesionales frente a la lectura: La lectura en España. p. 165209. En Informe 2008. Madrid: Federación de Gremios de Editores de España.

Cortez, F. (2006). Comunidades y trayectorias de lectura en la biblioteca pública: Un estudio etnográfico en el municipio de Chalco, Estado de México. Lectura y Vida, 27(1): 30-41. Obtenido de la base de datos EBSCOhost el 21 de agosto del 2011.

Davidovics, G. y Mayol, A. (2008). Introducción al uso de muestras para la realización de encuestas en la investigación social. P.7195. En: Salinas, P. y Cárdenas, M. (2008). Métodos de investigación social. Antofogasta: Ediciones Universidad Católica del Norte.

Domínguez, Mario. (2005). Transformaciones en las prácticas culturales de los jóvenes. De la lectura como ocio y consumo la fragmentación neotecnológica. Recuperado el 
19 de agosto del 2011 de http://dialnet.unirioja.es/servlet/articulo?codigo $=1387273$

Montilla, A. y Avalos C. (2012). Análisis de la encuesta de prácticas y hábitos culturales de Costa Rica, 2010-2011. Costa Rica: Dirección de Cultura del Ministerio de Cultura y Juventud. Recuperado el 27 de noviembre del 2012 de http://si.cultura. $\mathrm{cr} /$ images/documentos/indicadores/Primera_Encuesta/Primera\%20Encuesta\%20 Cultura.pdf

Nava Gómez, G., y García Ávila, C. (2009). Análisis de la cultura lectora en alumnos universitarios. Educación, 33(1): 41-59. Obtenido de la base de datos EBSCOhost el 19 de agosto del 2011.

Oficina de Registro e Información, Centro de Evaluación Académica y Vicerrectoría de Docencia (s.f). Hechos y cifras. Recuperado el 22 de agosto del 2011 de http://www. ucr.ac.cr/acerca-u/u-en-breve/hechos-cifra

Olaziregi, M.J. (2000). Aproximación sociológica a los hábitos de lectura de la juventud vasca. Oihenart, 18: 79-93.
Peredo, M., González R. (2007). Los jóvenes y sus lecturas: una temática común entre las revistas y los libros que eligen. RMIE, 33: 635-655. Obtenido de base de datos EBSCO Host el 19 de agosto del 2011.

Peña, F., Serrano, M. \& Aguirre, R. (2008). Prácticas de lectura en escolares: de la primera etapa de educación básica. Ducere investigación abitrada, No. 46. Recuperado el 20 de agosto del 2011 de http://www. saber.ula.ve/bitstream/123456789/31258/3/ articulo18.pdf.

Rockwell, Elsie. (2011). La lectura como práctica cultural: conceptos para el estudio de los libros escolares. Educação e Pesquisa, 27: 11-26. Recuperado el 18 de agosto de 2011 de http://dx.doi.org/10.1590/ S1517-97022001000100002.

Spang, K. (2000). Géneros Literarios. España: Editorial Síntesis.

Vílchez, Carlos M. (2003). Hábitos de lectura de los adolescentes peruanos. Revista Interamericana de Bibliotecología, 26, 1-19. 